Website: http://jsci.utq.edu.iq

Email: utjsci@utq.edu.iq

تأثثر نسجة التربة وكمية الماء المضافة في حركة الماء في مقد التربة المروية بالتنقيط

$$
\begin{aligned}
& \text { قيصر ابر اهيم حمد } \\
& \text { مركز أبحاث الاهو ار - جامعة ذي قار } \\
& \text { الخلاصــة }
\end{aligned}
$$

أجريت الدراسة لمعرفة ناثير كمية الماء المضافة للتربة على حركة الماء افقبا وعموديا لتربتين مزيجية رملية (S1)

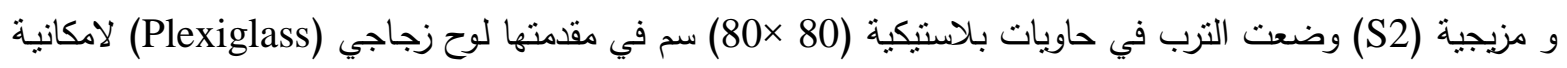

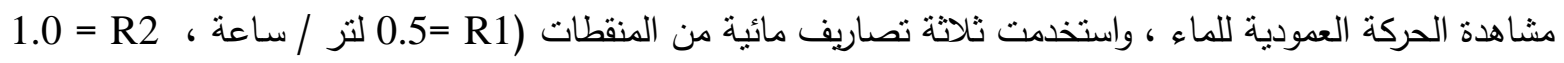

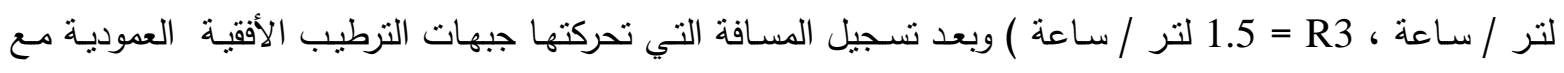

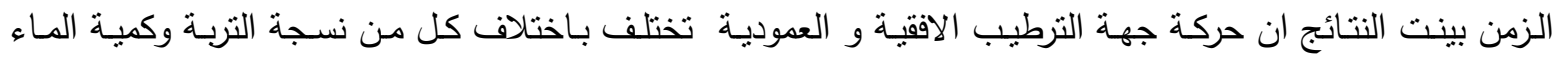

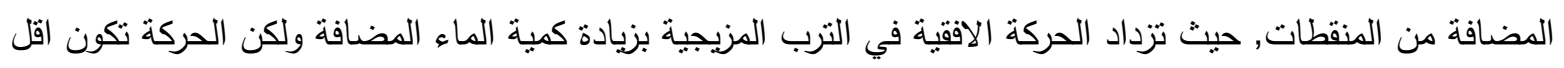

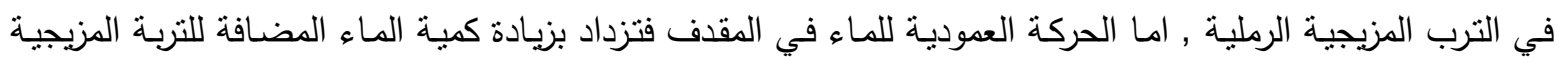
الرملية عنها في الترب المزيجية ولكن في نهاية القياس فان الحركة العمودية تكون اكبر من الافقية في كلتا التها التربتين

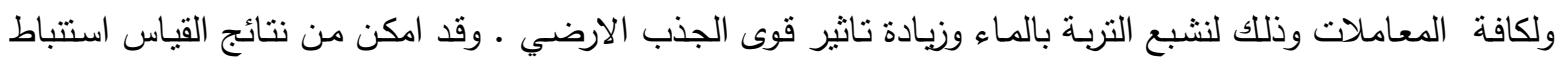

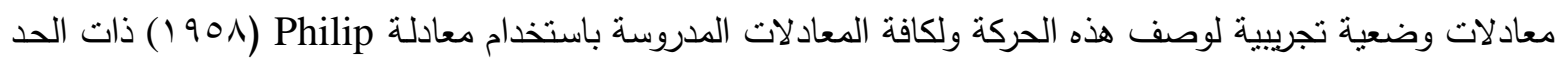

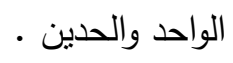

\title{
Effect of soil texture and water application rate on water movement in soil profile under trickle irrigation
}

\section{ABSTRACT}

the study was conducted to know the effect of water application rate $(\mathrm{R})$ and soil texture (S) on vertically and horizontally movement of water in the soil profile under triclcle irrigation two type of soil texture (S1 sandy Loam, S2 Loamy soil ) and three water application rate $(\mathrm{R} 1=0.5 \mathrm{~L} / \mathrm{h}, \mathrm{R} 2=1.0 \mathrm{~L} / \mathrm{h}$ and $\mathrm{R} 3=1.5 \mathrm{~L} / \mathrm{h})$ was used.The used soil was in plastic container( $80 * 80) \mathrm{cm}$ with plexiglass in one side of container to show the vertically movement of water clearly. The results were showed that the horizontal wet front increases with increasing the water application rate in the Loam soil as compard with sandy loam soil, but the vertically distance of wet front at the sandy loam was more than the same distance at the loam soil . The Philip (1958) one and two term equation used to fit the experimental data of wet front distance with time.Emperical equation that describe this movement were also used for the all treatment. 
هذه الحركة ـ واوضـح كل من (£) و (9 ()) بـان الحركة الاققية والعمودية للماء في مقد التزبة هي دالة لكمية الماء المضاف ونوع التربة في حين اشار (؟) بان هناك الكثير

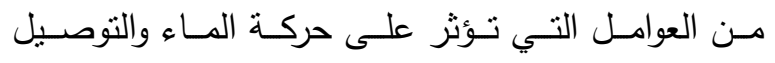
الهيدروليكي كنسجة التربة والمواد اللاحمة لاقائقها وكمية المـاء المضـاف وان الحجم النهائي للمنطقة المبللة اسفل

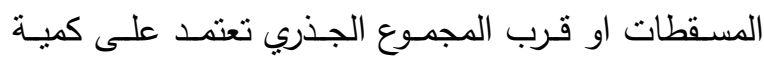
الماء المضافة ونسجة التزبة ـ تهدف الدراسة لمعرفة تاثير نسجة التربة وكمية الماء المضافة اليهاعلى الحركة الافقية

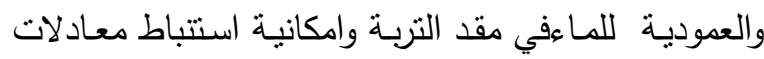

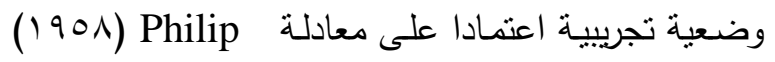

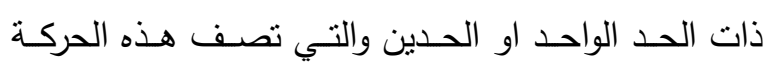
لامكانية معرفة الزمن اللازم لتشغيل منظومة الري بالتتقيط للوصول الى كمية رطوبة ملائدة لنمو النبات دون الهدر في استخدام المياه . المو ادوطرق العمل اجريت الدراسه في الظله الخشبية التابعه لمحطة بستته

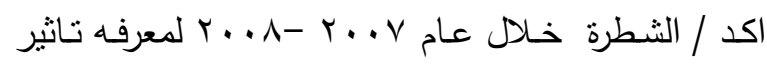

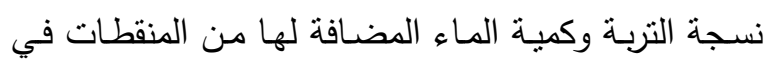
حركة الماء افقيا وعموديا في مقدها .

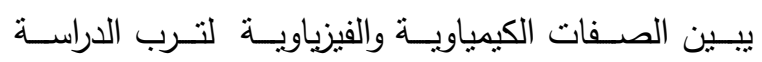

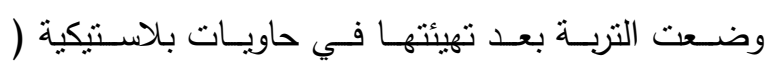
سنادين) (80 ×80) سم عدا جانب واحد وضعت علية شريحة زجا جية (Plexiglass) لاككانية مشاهدة الحركة

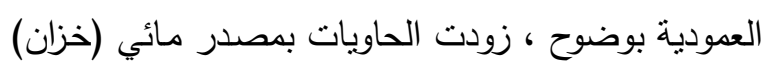

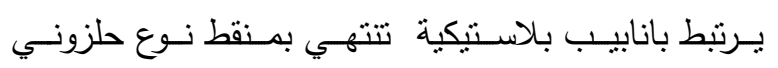

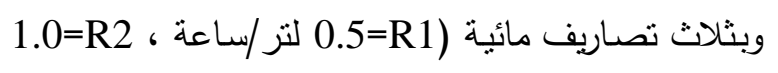

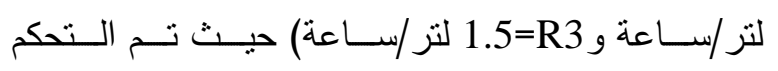

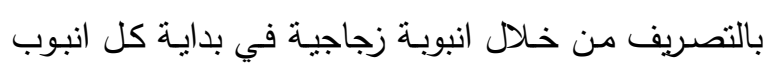

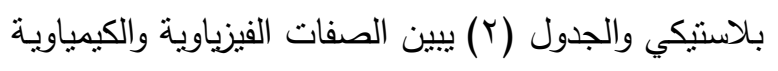

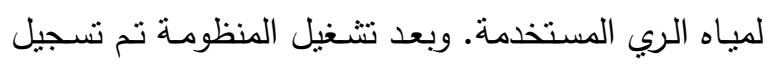

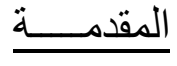
ان استغلال الموارد المائية علميا واقتصاديا من الاهمية

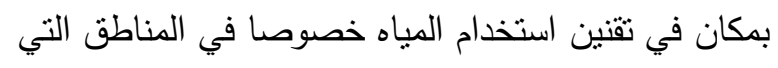

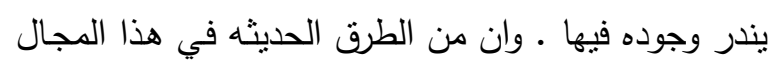
هما طريقتي الري بالرش والتتقيط والتي ينطلب استخدامها

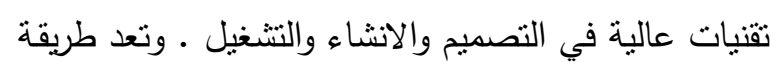

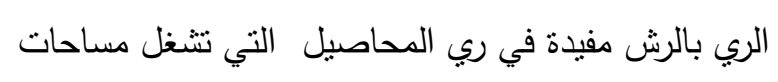

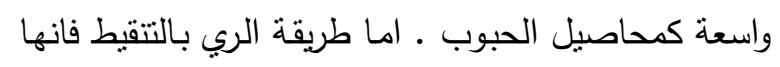

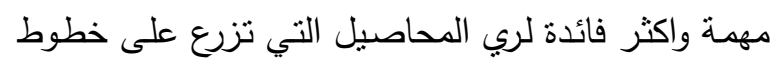

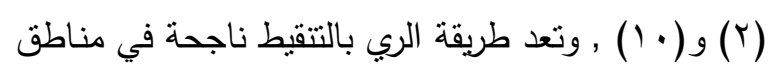
معينة من العراق التي يصعب استخدام الطرق الاخرى فيها

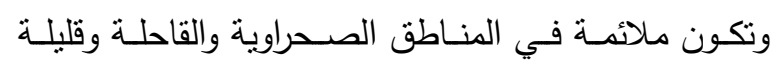

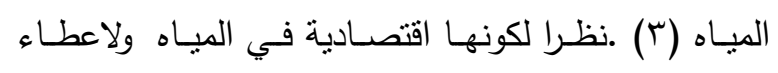

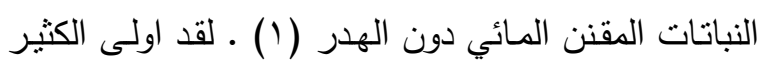

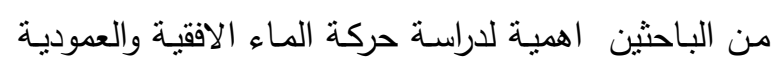

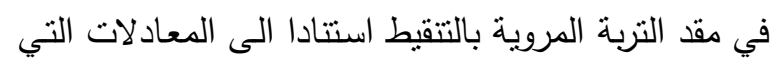

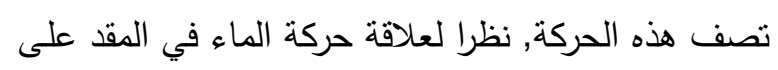

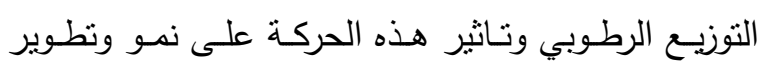

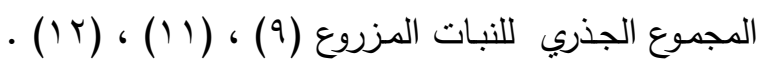

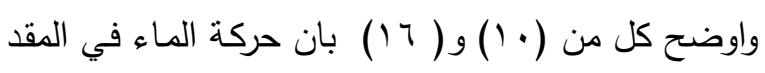

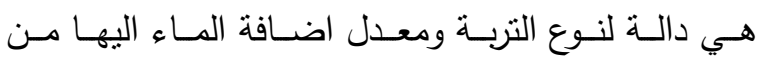

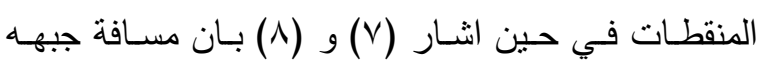

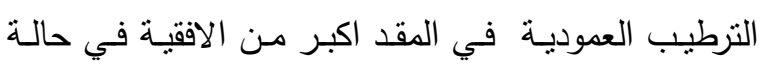

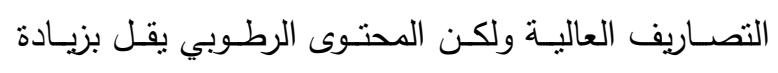

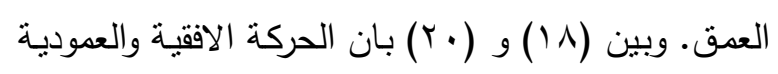

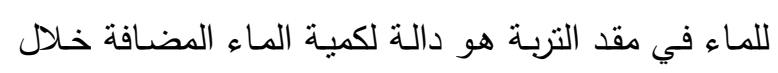

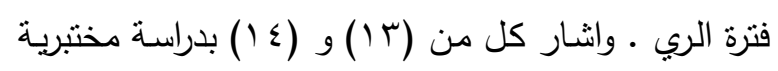
لتربتين مختلفتي النسجة وتحت معدلات مختلفة من الماء

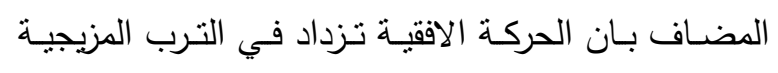

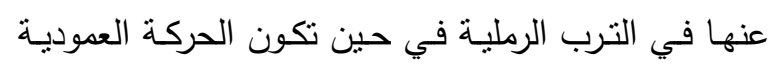

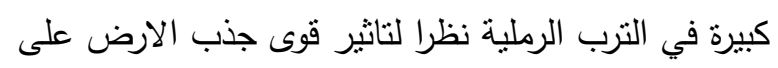




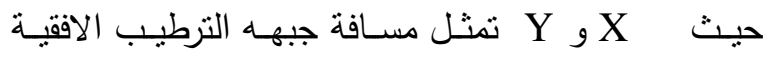

والعمودية على التوالي بوحدات (L)

N ميـل المسـتقيم المرسـوم للعلاقـة بـين مسـافة جبهـة

الترطيب الافقية (X) مع الزمن (T) مئمن

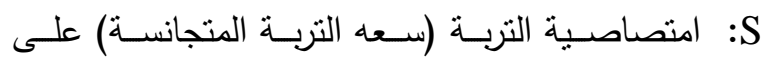

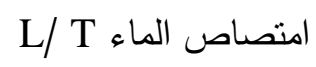

:A انتشارية التربة (قدرة التربة على توصيل الماء) وتعتمد على الصفات المائية وجهه الجذب الارضي.

T
المسـافة التـي تحركتهـا كـل مـن جهـه الترطيـب الافقيـة والعموديـة مـع الزمن ـ قدرت الايصـالية الكهربائية ودرجـة التفاعـل فـي عجينــة التربــة المثــبعة والمــاء بجهــاز Professional PP 50 Multiparameter والانيونـات للتربـة والمـاء بجهاز

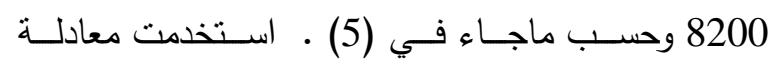

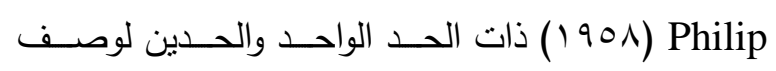
الحركة الافقية والعمودية على التوالي وكما يلي :-

$$
\begin{aligned}
& X=N^{2 / 1} t \\
& Y=S^{2 / 1} t+A t
\end{aligned}
$$

\begin{tabular}{|c|c|c|c|}
\hline تربة مزيجية & تربة مزيجية رملية & الصفة & \\
\hline 4,21 & 3,46 & Fلمي موز/سم) & التوصيل الكه \\
\hline 8,62 & 8,38 & \multicolumn{2}{|c|}{ درجة التقاعل pH } \\
\hline 6,85 & 16,92 & & الكالمبوم \\
\hline 5,91 & 4,12 & & المقتيسيوم \\
\hline 16,01 & 13,34 & & الصوديوم \\
\hline 1,12 & 0,35 & ح ملي مكافي/لتزر & البوتلسيوم \\
\hline 1.64 & صغر & & الكاربونات \\
\hline صفر & 1,23 & & اليبكاريونات \\
\hline 14,25 & 17,01 & & الكبريتات \\
\hline 13,87 & 14,23 & & الكلورايد \\
\hline 41,29 & 65,29 & & الرمل \% \\
\hline 30,37 & 15,41 & & الغرين \% \\
\hline 28,34 & 19,30 & & الطين \\
\hline
\end{tabular}

جدول (1) الصفات الفيزياوية والكيمياوية لترب الدراسة 


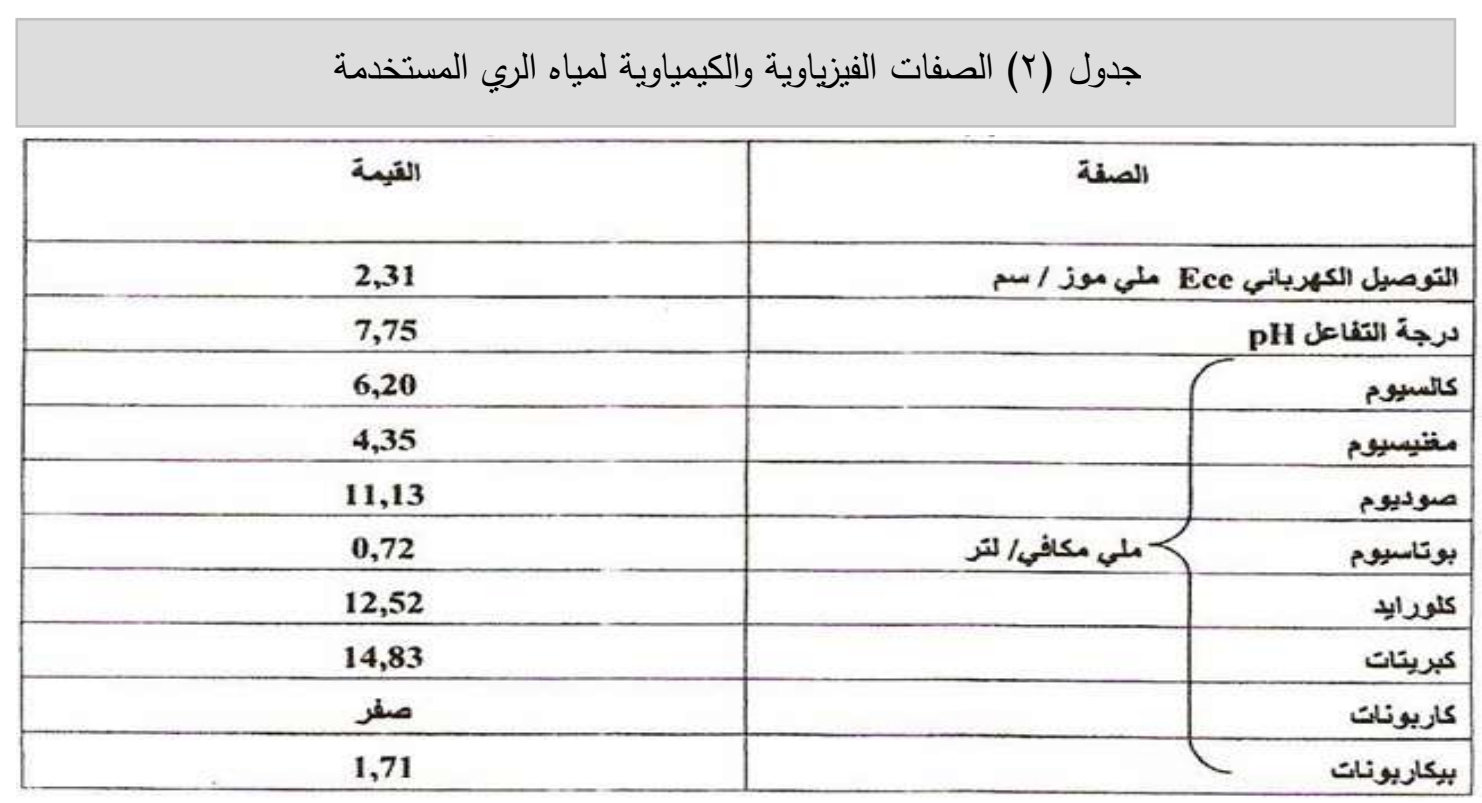

اوضحو بان المسافة الني تتحركها جبهه الترطيب الافقية

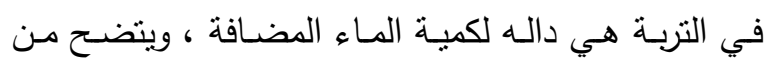

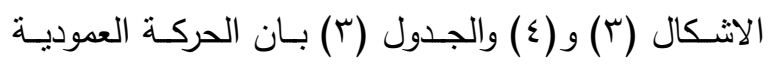

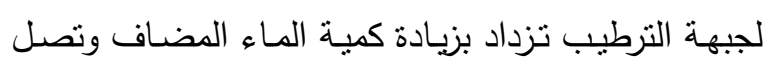

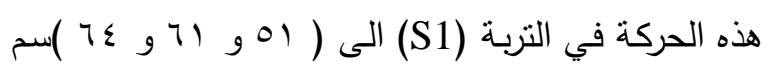

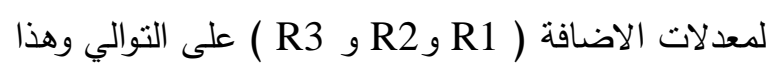

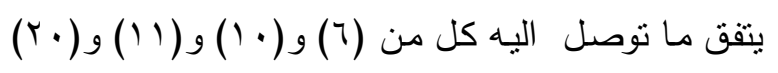

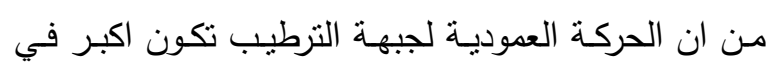

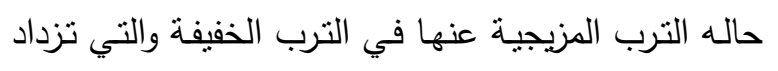

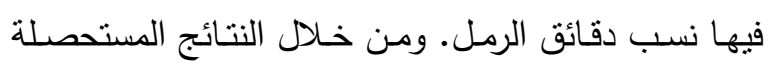

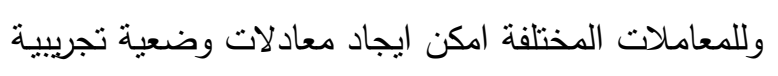
باستخدام برنامج الارتداد اللاخطي وذلك لمطابقة النتائج

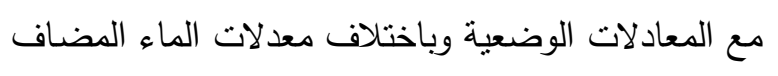

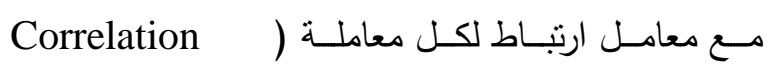
Standarddeviation (Coeffirient

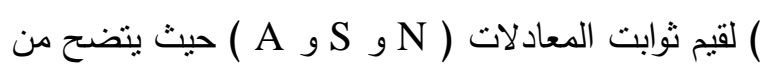
الجدول (؟) بان المعامل (N) في التربة الخشنة النسجة

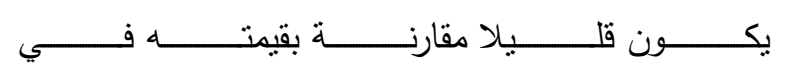

\begin{abstract}
النتائج و المناقثة بيين الجدول ( (1) و (r) الصفات الفيزياوية والكيمياوية

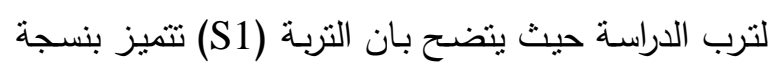

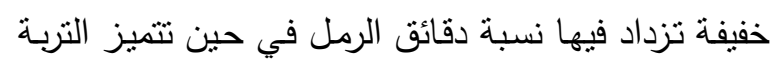

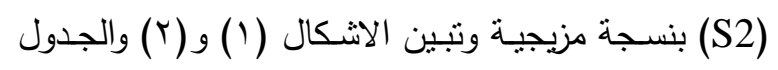

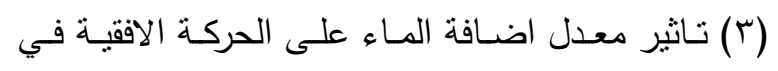
قطاع التربـة (S1) اذ يلاحظ بان المسافة التي تتحركها

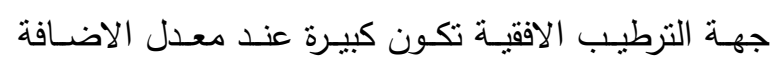

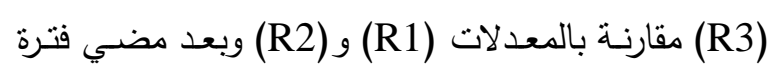
قصيرة تصل الحركة الاقفية لجهه الترطيب الى قيم قليلة

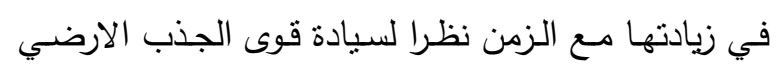

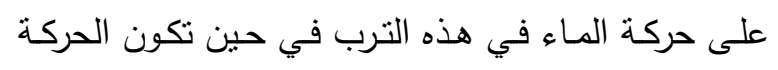

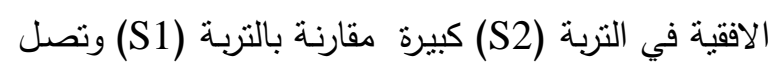
مسافة جهه الترطيب الافقية على جانبي مصدر الاضافة

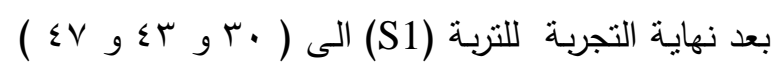

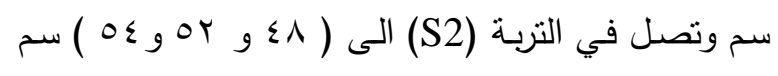
لمعدلات المساء ( R1 و R2 و R3 ) على التى التوالي وهذه

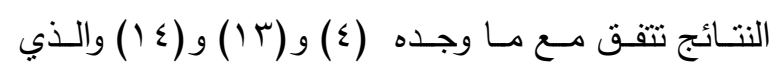


لنموه من خلال معرفة عمق المجموع الجذري والذي يمتله

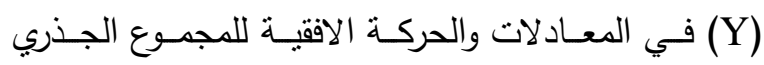

$$
\text { للنبات والذي يمثله (X) }
$$

يوصـي الباحـث بـاجراء الدراســات باسـتخدام تـرب ذات نسجات مختلفة وكميات مياه مختلفه لنبات لمعرفة تاثير النسـجة ومعـدل المـاء المضـاف على حركـة المـاء افقيـا

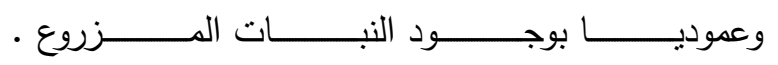

الترب الناعمـة النسـجة كمـا ان المعامـل (S) والذي يمثل

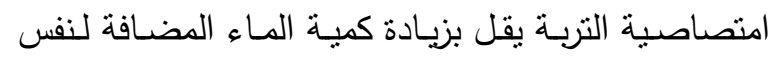
التربة في حين يزداد المعامل (A) والذي يمثل انتشار ماء التربة بزيادة كمية الماء المضـاف ولكافة المعاملات ومن لهن خـلال المعـادلات التجريبيـة التـي امكـن الحصـول عليهـا ولنفس ظروف الترب المستخدمة في الدراسة يمكن حساب فتراة الري اللازمة من خلال معرفة الزمن الـازم للوصول

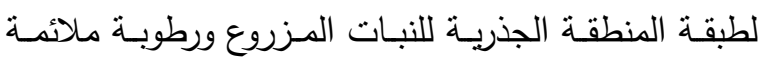

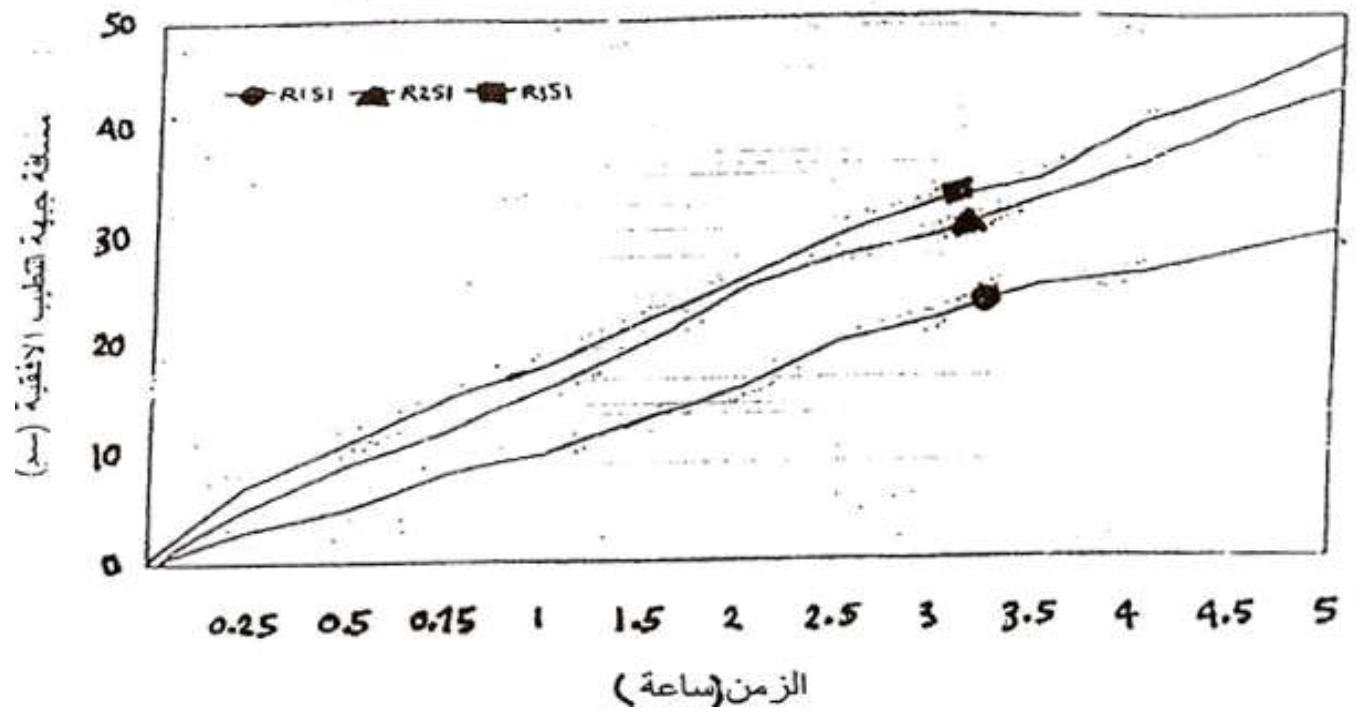

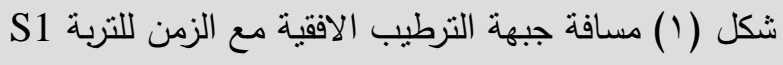

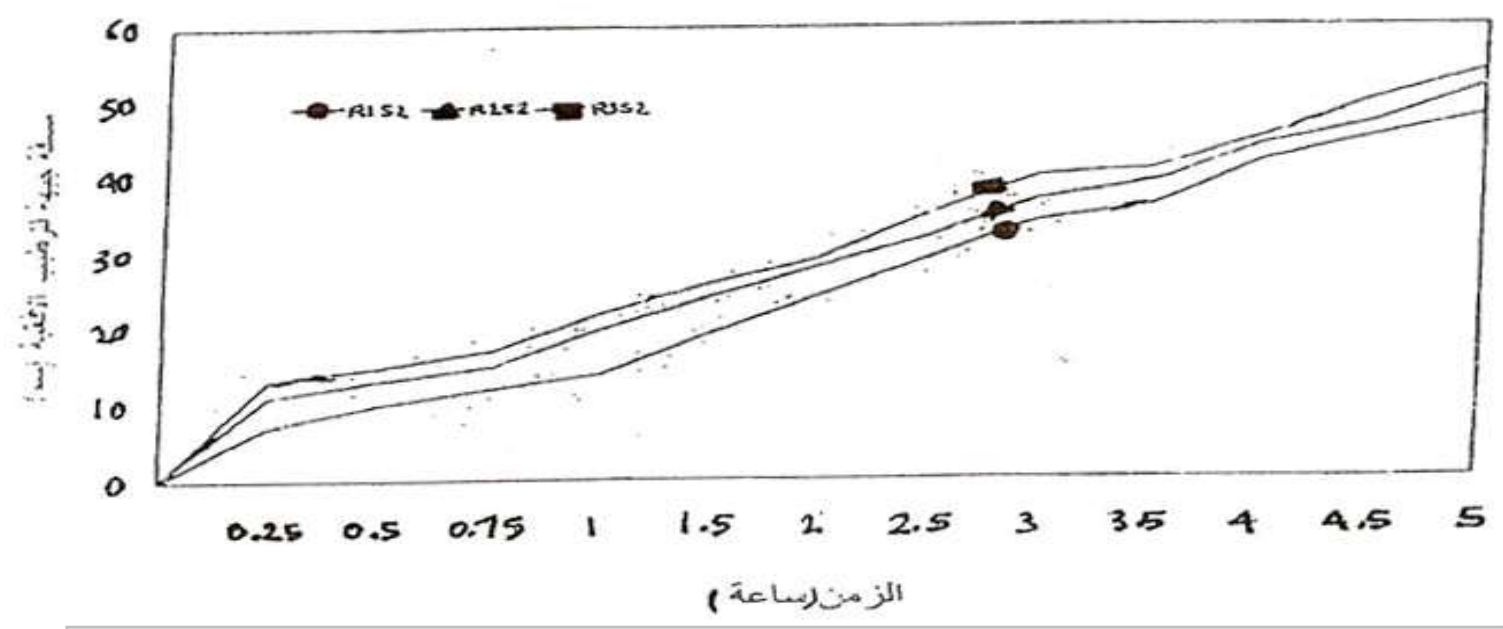

S2 شكل (r) مسافة جبهة الترطيب الافقية مع الزمن للتربة 


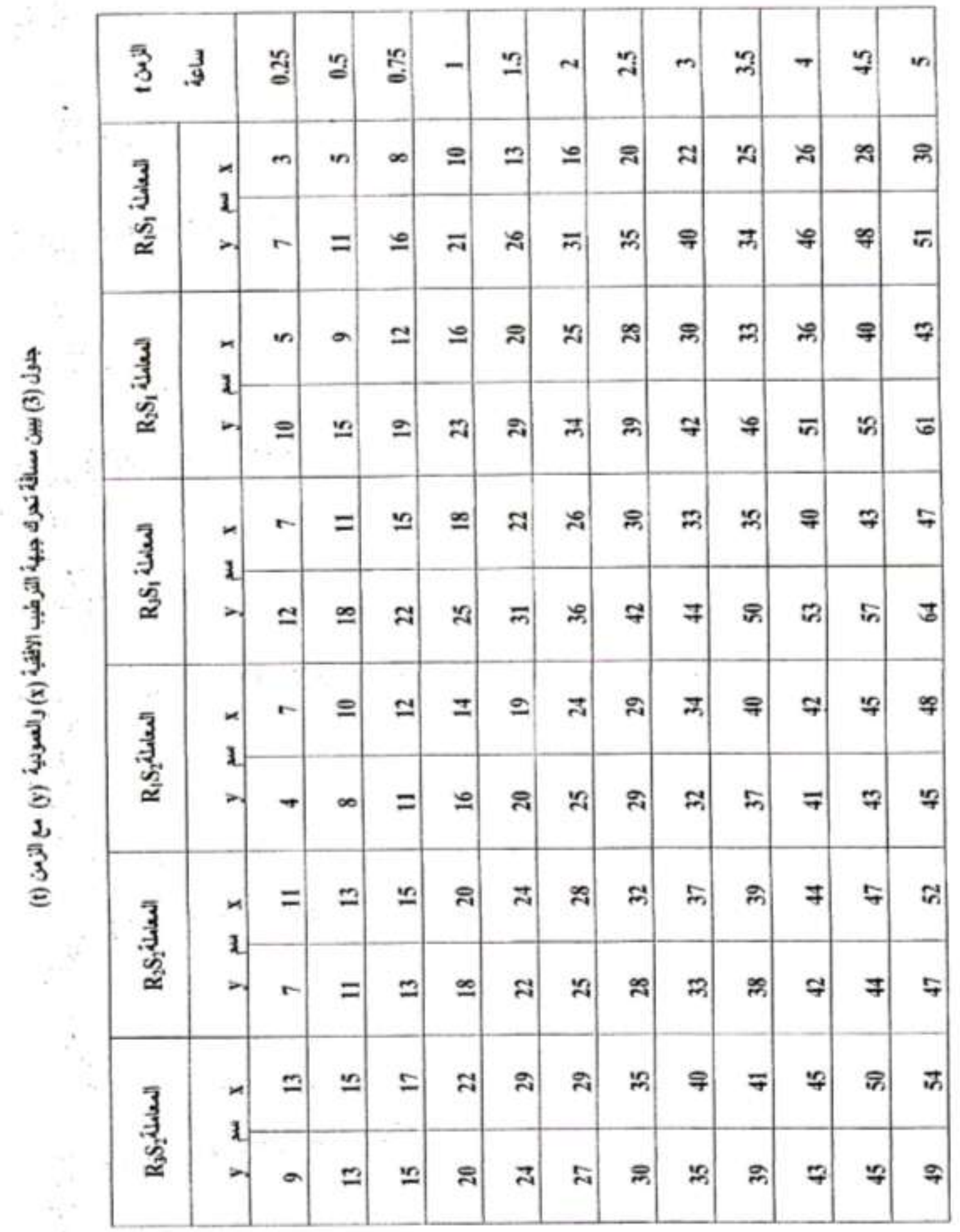




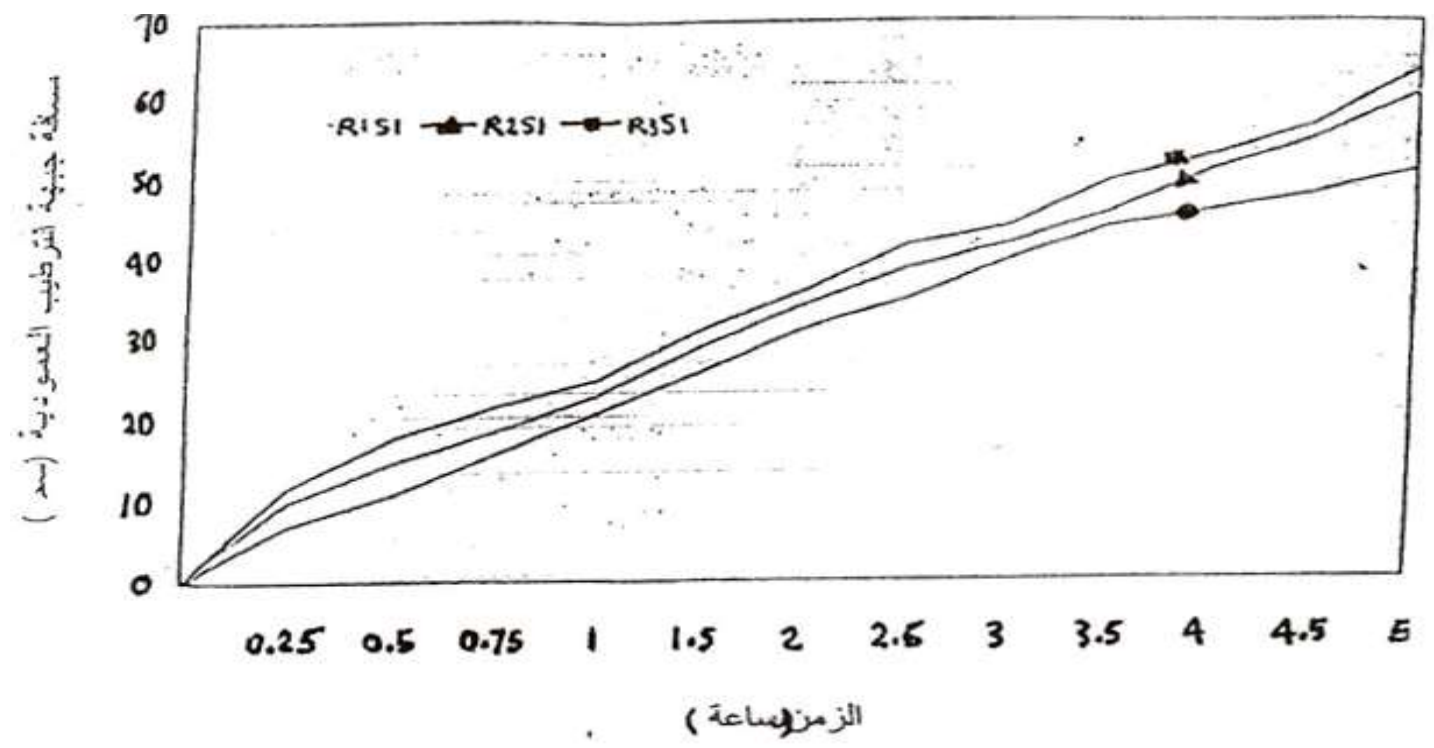

S1 شكل (r) مسافة جبهة الترطيب العمودية مع الزمن للتربة

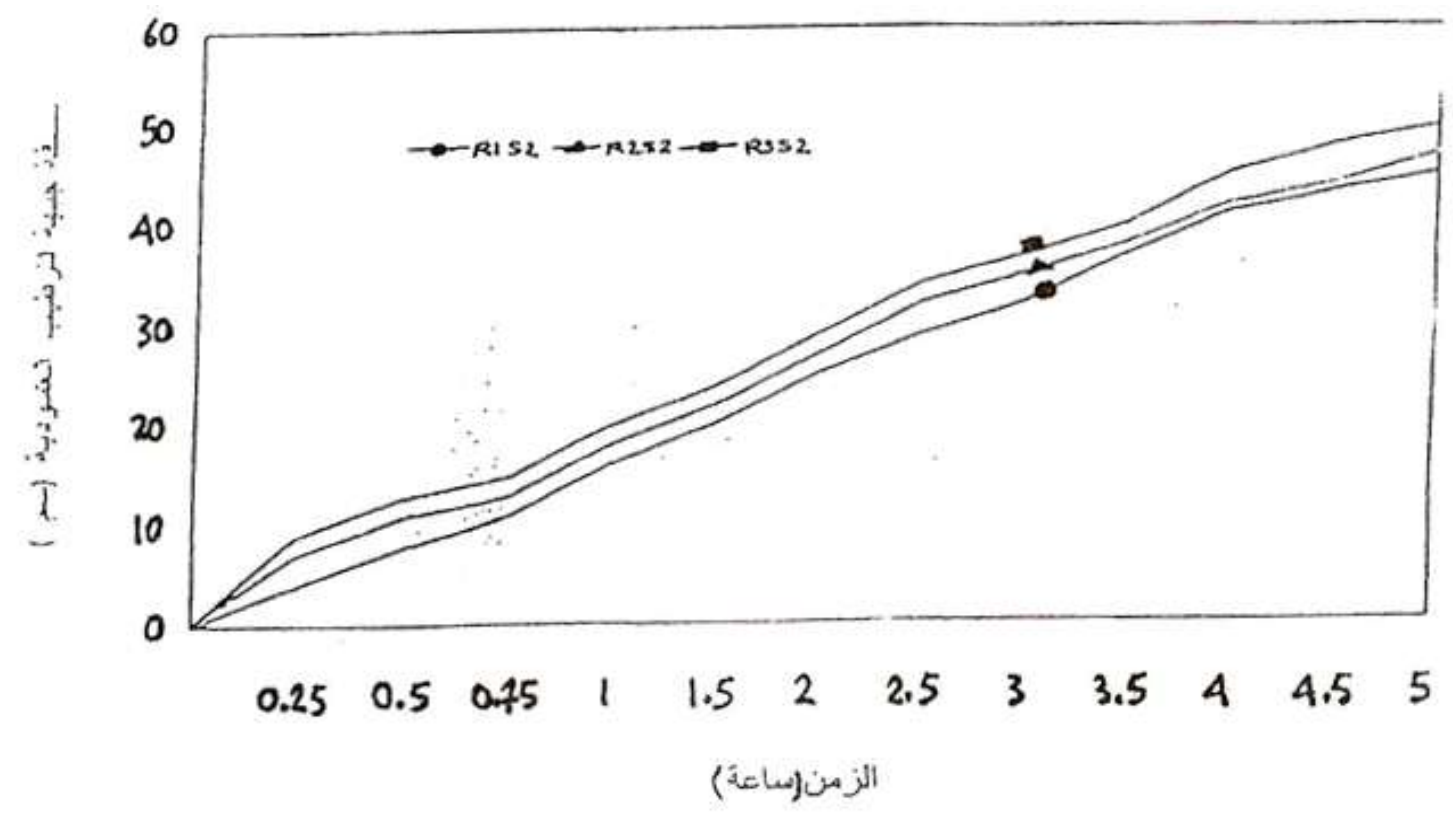

S2 شكل (ع) مسافة جبهة الترطيب العمودية مع الزمن للتربة 


\section{تموز/|}

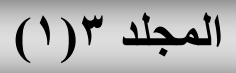

مجلة علوم ذي قار

جدول (4) المسادلات الوضسية للمعاسلات المختلفة

\begin{tabular}{|c|c|c|c|}
\hline المعاملة & 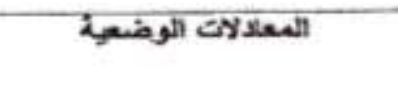 & 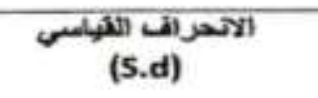 & 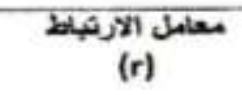 \\
\hline $\mathrm{R}_{\mathbf{1}} \mathrm{S}_{\mathbf{1}}$ & $\begin{array}{c}x=1.9 t^{\frac{1}{2}} \\
y=13.1 t^{\frac{1}{2}}+6.1 t\end{array}$ & $\begin{array}{l}\text { S. } d N \mp 1.012 \\
\text { S. } d S \mp 1.240 \\
\text { S. } d a \mp 1.152\end{array}$ & $\begin{array}{l}0.852^{*} \\
0.877^{*}\end{array}$ \\
\hline $\mathrm{R}_{2} \mathrm{~S}_{1}$ & $\begin{array}{c}x=17.8 t^{\frac{1}{2}} \\
y=12.4 t^{\frac{1}{2}}+6.5 t\end{array}$ & $\begin{array}{l}\text { S. } d N \mp 1.330 \\
\text { S. } d S \mp 1.211 \\
\text { S.d a } \mp 1.412\end{array}$ & $\begin{array}{l}0.891^{\cdots} \\
0.894^{-2}\end{array}$ \\
\hline $\mathrm{R}_{3} \mathrm{~S}_{1}$ & $\begin{array}{c}x=19.7 t^{\frac{1}{2}} \\
y=11.5 t^{\frac{1}{2}}+7.3 t\end{array}$ & $\begin{array}{l}\text { S. } d N \mp 1.089 \\
\text { S. } d S \mp 1.035 \\
\text { S. d } a \mp 1.112\end{array}$ & $0.912^{*-}$ \\
\hline $\mathrm{R}_{1} \mathrm{~S}_{2}$ & $\begin{array}{c}x=20.5 t^{\frac{1}{2}} \\
y=11.8 t^{\frac{2}{2}}+5.8 t\end{array}$ & $\begin{array}{l}\text { S. } d N \mp 1.215 \\
\text { S. } d S \mp 1.314 \\
\text { S. } d a \mp 1.046\end{array}$ & $\begin{array}{l}0.854^{-} \\
0.899^{-2}\end{array}$ \\
\hline $\mathrm{R}_{2} \mathrm{~S}_{2}$ & $\begin{array}{c}x=21.8 t^{\frac{1}{2}} \\
y=10.7 t^{\frac{1}{2}}+6.4 t\end{array}$ & $\begin{array}{l}\text { S. } d N \mp 1.255 \\
\text { S. } d S \mp 1.301 \\
\text { S. } d a \mp 1.244\end{array}$ & $\begin{array}{l}0.896^{*-} \\
0.882^{*}\end{array}$ \\
\hline $\mathrm{R}_{3} \mathrm{~S}_{2}$ & $\begin{array}{c}x=23.0 t^{\frac{2}{2}} \\
y=10.3 t^{\frac{1}{2}}+6.9 t\end{array}$ & $\begin{array}{l}\text { S. } d N \mp 1.095 \\
\text { S. d } S \mp 1.089 \\
\text { S.d } a \mp 1.101\end{array}$ & $0.911^{-x}$ \\
\hline
\end{tabular}


14-Hachum , A.Y; J.F. Alfaro and L.W. Willardsomes (1973) Water movement in soil from drain .Div. Am.Soc .Agric . Eng . 102(1122):179-192.

15-Jackson . M.L (1958).Soil chemical analysis . Englwood clift . N.J.U:S.A.

16-Joseph , A.K. and R.A. Laipet . (2005) Infiltration and redistribution of water across soil textural classes Soil Sci . Am .J. 69:816-829 .

17-Philip , J.R . (1958) .The theory of Infiltration . Soil Sci. J. 55:333-337 .

18-Roth , R.L. (1974). Soil moisture and wetting pattern from point source. Proc . second int . Drip irrigation congress, San Diego . California P:246-251 .

19-SmaJstrla , A.Q. and S.L.Locascio. (1994) . Tensiometer controlled drip shedulling of tomato, An ASAE meeting presentation . Pp. 94.2588.Atlanta . USA

20-Toma kazoo , F . and I . munchide . (2008). Upward Infiltration into porous as affected by wetability and anionic surfactant . Soil Sci . Am . J. 72:741-749

$$
\begin{aligned}
& \text { محصول الطماطة المزروعـه تحت نظام الري بالتتقيط . } \\
& \text { اطروحة دكتوراه ، كلية الزراعة ، جامعة البصرة ، العراق } \\
& \text { r- محمد كامل مجيد (ع 911) ـ الري بالتنقيط ـ وزارة الري ، } \\
& \text { المنشاة العامة للواحات الصحراوية وصيانة التربة ، بغداد }
\end{aligned}
$$

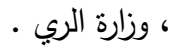

4-Amen ,W.R.(1981).Water movement and distribution in soil of northern in Iraq under trickle source. M.Sc. thesis . College of Eng . un of Mosul, Mosul, Iraq .

5-Black, G.R. (1965) . (Bulk) Density . inc . A. Black et al . (eds). Methods of soil analysis . Part 1 . Am . Soc . Agron . $\mathrm{P}: 377-381$.

6-Bosch, D.D. and West L.T. (1998). Hydraulic conductivity variability for two sandy soils . Soil Sci . Am.J.62:90-98

7-Brandt,A.E;Bresler , N.D ; I. Ben-Asher ; J. Heller and Goldberg (1971). Infiltration from trickle source. Mathematical Method . Soil Sci. Am .J. 35:675-682

8-Bresler , E . (1975) . Tow dimentional transport of solutes during non - steady infiltration from triclcle source. Soil Sci . Am . J. 39: 604-612.

9-Degong , L.W;O.H. Jacobson , P. Moldrup (1999). Effect of water content , temperature, and size of particles on soil water repellency Soil Sci .Am . J.63:437442.

10-Goldberg , D.and M.Shmeuli (1970).Drip irrigation A: Method used under arid and desert condition of high water and soil salinity. Trans . Am. Soc .Agr . Eng . $\mathrm{P}: 38-41$.

11-Goldberg, D.B.Gormat and R.Bar . (1970) a. The disitribution of roots, water and minerals as aresult of triclcle irrigation. Am.Soc. Hort . Sci.J. 96:645-684 .

12-Goldberg, D.M.R.and N.Karn (1971). Effect of irrigation intervals on distribution and utitization of soil moisture in vineyard . Soil Sci . Am. Proc . 3:127-130.

13-Hachum , A.Y. (1973). Water movement in soil from trickle source. M.Sc. thesis . Utah state university, Lodan . Utah . 